Research Article

\title{
Singularity Analysis of Redundant Space Robot with the Structure of Canadarm2
}

\author{
Gang Chen, Long Zhang, Qingxuan Jia, and Hanxu Sun \\ School of Automation, Beijing University of Posts and Telecommunications, Beijing 100876, China \\ Correspondence should be addressed to Gang Chen; buptcg@gmail.com
}

Received 1 March 2014; Revised 21 May 2014; Accepted 21 May 2014; Published 26 June 2014

Academic Editor: Alessandro Palmeri

Copyright (C) 2014 Gang Chen et al. This is an open access article distributed under the Creative Commons Attribution License, which permits unrestricted use, distribution, and reproduction in any medium, provided the original work is properly cited.

A novel method of singularity analysis for redundant space robot with the structure of Canadarm2 is proposed in this paper. This kind of structure has the characteristics of three consecutive parallel axes. First, the "virtual manipulator" method is employed to transfer the singularity problem of a space robot to that of a ground one. By choosing an appropriate reference system and a reference point of the end-effector, Jacobian matrix is greatly simplified and then it is reconstructed according to a new standard. On this basis, the Jacobian matrix can be partitioned into four submatrixes whose degradation conditions are put forward; thereafter, the singularity conditions and singular directions of the redundant space robot are obtained. The effectiveness of the proposed singularity analysis method is verified through simulation.

\section{Introduction}

Singularity is the inherent characteristic of robots. At a singular configuration, the end-effector of the robot loses the ability to move along a certain direction, which is called the singular direction. In this case, if there is still a velocity component of the end-effector in singular direction, the joint angular velocities will become unacceptably large, and the end-effector will deviate from its expected trajectory. Moreover, it is possible that a robot will be out of control $[1,2]$. Therefore, in order to ensure the stability and reliability of robot, it is necessary to do the research on singularity analysis.

Robot singularity analysis, as the basis of singularity avoidance, is used to determine the singularity conditions. For nonredundant robots, the determinant value of Jacobian matrix can directly determine robot singularity conditions. However, for redundant robots, because its Jacobian matrix is not square, the singularity analysis becomes much more complex. Much effort in research community has been paid on dealing with singularity analysis of redundant robots. Whitney [3] determined singularity conditions by calculating the determinant value of matrix $\mathrm{JJ}^{\mathrm{T}}$. The pseudoinverse of Jacobian matrix $\mathbf{J}$ is given by $\mathbf{J}^{\dagger}=\mathbf{J}^{\mathrm{T}}\left(\mathbf{J}^{\mathrm{T}}\right)^{-1}$. Singular configurations occur when the determinant value of the $\mathrm{JJ}^{\mathrm{T}}$ portion of $\mathbf{J}^{\dagger}$ is equal to zero. Although the matrix formed by $\mathrm{JJ}^{\mathrm{T}}$ is a square matrix, the determinant expression could be quite complicated, making it very difficult to obtain the analytical solution. Chou et al. [4] defined the concept of null motion differential dynamic system, and then the local topology and singularity avoidability of redundant robots are studied according to nonlinear singularity theory. Podhorodeski et al. [5] proposed using six-joint subgroups of Jacobian matrix to determine the singularity conditions of redundant robots. Conditions that cause the determinant values of all possible six-joint subgroups to simultaneously equal zero are singularity conditions. The method works well, but the entire solving process is much complicated. Nokleby and Podhorodeski $[6,7]$ used the properties of reciprocal screws to determine the single-DOF-loss and the multi-DOF-loss conditions of redundant robots and also work out the singular directions. This method is also used in the singularity analysis of Canadian Space Agency (CSA) Canadarm2 [8]. The reciprocal method is effective for the singularity analysis of all redundant serial manipulators. However, with the increase of DOF, its calculation amount is little large. For a 7-DOF robot with the structure of the last, three axes perpendicular to each other, Waldron et al. $[9,10]$, based on matrix partitioning, converted 
the segmented $3 \times 4$ submatrix to a $4 \times 4$ submatrix with an additional relationship between joint angular velocities. And then the singularity conditions can be achieved through the determinant value of square submatrixes. Due to the introduction of an additional relationship, the method may encounter algorithm singular problems. Cheng et al. [11] suggested decomposing singularities into position singularities and orientation singularities by partitioning the $6 \times$ 7 Jacobian matrix. This method only needs to determine each singularity condition of the submatrixes including two $3 \times$ 4 matrixes, one $3 \times 3$ matrix, and one $3 \times 3$ zero matrix to obtain the singularity conditions. It can greatly reduce the computation complexity, but it can only be applied to the singularity analysis of the 7-DOF robots with the structure of the last three axes perpendicular to each other.

All the singularity analysis methods mentioned above are aiming at ground robots or space robots in fixed-base mode. For free-floating or free-flying space robots, some scholars utilized the method in [2], only replacing the Jacobian matrix $\mathbf{J}$ by $\mathbf{J}_{g}$ in $\mathbf{J}^{\dagger}=\mathbf{J}^{\mathrm{T}}\left(\mathbf{J} \mathbf{J}^{\mathrm{T}}\right)^{-1}$; here, $\mathbf{J}_{g}$ is called the generalized Jacobian matrix. Similarly, it is very difficult to obtain the analytical solution. Xu et al. [12] presented a singularity separation method which separates the singularity parameters from the inverse of the Jacobian to simplify the analysis. However, sometimes the parameters separation is hard. Nenchev et al. [13] utilized the singular-value decomposition (SVD) of the Jacobian matrix to judge whether the singularity happens, and it is really effective for numerical computation not for analytical solution.

A novel method of singularity analysis for redundant space robots with the structure of Canadarm 2 is proposed in this paper. First, the "virtual manipulator" method is employed to transfer the singularity problem of a space robot to that of a ground one. Second, Jacobian matrix is reconstructed according to a new standard on the basis of simplification. Thereafter, the Jacobian matrix can be partitioned into four submatrixes whose degradation conditions are put forward. Finally, the singularity conditions and singular directions of redundant space robots with the structure of Canadarm2 are obtained.

This paper is organized as follows. Section 1 gives a brief overview of the research on singularity analysis. Section 2 employs the "virtual manipulator" method to transfer the singularity problem of a space robot to that of a ground one. The Jacobian matrix is simplified and the equivalence of singularity is analyzed in detail in Section 3. Section 4 obtains the singularity conditions and singular directions. Section 5 is a simulation example and Section 6 concludes the paper.

\section{Virtual Manipulator}

For a free-floating space robot as shown in Figure 1, the linear momentum of the system is conserved, which is the holonomic constraint. According to "virtual manipulator" concept proposed in $[14,15]$, the kinematic problem in this mode can be simplified to that belonging to a ground robot. By this concept, the base of free-floating manipulator is regarded as a rod linked to the virtual ground by a passive sphere joint. The relationship between system mass center and the each link's mass center is as follows (without special instruction, all variables are expressed in inertial frame):

$$
M r_{g}=\sum_{i=0}^{n} m_{i} r_{i}
$$

And the base's mass center is

$r_{0}=r_{g}-\frac{\left(m_{1}+\cdots+m_{n}\right)\left(b_{0}+a_{1}\right)}{M}-\cdots-\frac{m_{n}\left(b_{n-1}+a_{n}\right)}{M}$.

The position of the end-effector is

$$
p_{e}=r_{g}+\widehat{b}_{0}+\sum_{i=1}^{n}\left(\widehat{a}_{i}+\widehat{b}_{i}\right)
$$

where $\widehat{a}_{i}=\left(\left(\sum_{k=0}^{i} m_{k}\right) / M\right) a_{i}, \widehat{b}_{i}=\left(\left(\sum_{k=0}^{i} m_{k}\right) / M\right) b_{i}, i=$ $1, \ldots n$. Vectors $\widehat{a}_{i}$ and $\widehat{b}_{i}$ are called "virtual link vectors," which are parallel to vectors $a_{i}$ and $b_{i}$, respectively.

Differentiating two sides of (3), (4) can be obtained as follows:

$$
v_{e}=v_{g}+\dot{\vec{b}}_{0}+\sum_{i=1}^{n}\left(\dot{\hat{a}}_{i}+\dot{\hat{b}}_{i}\right)
$$

where $v_{g}$ represents the linear velocity of the system's mass center. Assuming that the initial momentum is zero, $v_{g}=$ 0 can be obtained due to the holonomic constraint. Moreover,

$$
\begin{aligned}
\dot{\vec{a}}_{i} & =\frac{d\left(\widehat{a}_{i}\right)}{d t}=\frac{d\left(\mathbf{A}_{i}{ }^{i} \widehat{a}_{i}\right)}{d t}=\frac{d\left(\mathbf{A}_{i}\right)}{d t}\left({ }^{i} \widehat{a}_{i}\right) \\
& =\left(\omega_{i} \times \mathbf{A}_{i}\right)\left({ }^{i} \widehat{a}_{i}\right)=\omega_{i} \times \widehat{a}_{i} .
\end{aligned}
$$

In the same way,

$$
\dot{\widehat{b}}_{i}=\omega_{i} \times \widehat{b}_{i}
$$

where $\mathbf{A}_{i}$ is the rotation matrix from frame $i$ to the inertial frame and $\omega_{i}$ is the angular velocity of $i$ th body, and combining (4), (5), and (6),

$$
v_{e}=\omega_{0} \times \widehat{b}_{0}+\sum_{i=1}^{n} \omega_{i} \times\left(\widehat{a}_{i}+\widehat{b}_{i}\right)
$$

Meanwhile, the angular velocity of link $i$ is

$$
\omega_{i}=\omega_{0}+\sum_{k=1}^{i} k_{k} \dot{\theta}_{k}
$$

Substituting (8) into (7),

$$
v_{e}=-\widetilde{\mathbf{p}}_{g e} \omega_{0}+\sum_{k=1}^{n}\left[k_{k} \times\left(p_{e}-\widehat{p}_{k}\right)\right] \dot{\theta}_{k}
$$

where $\widetilde{\mathbf{p}}_{g e}$ is the antisymmetric matrix of vector $\left(p_{e}-r_{g}\right)$. 


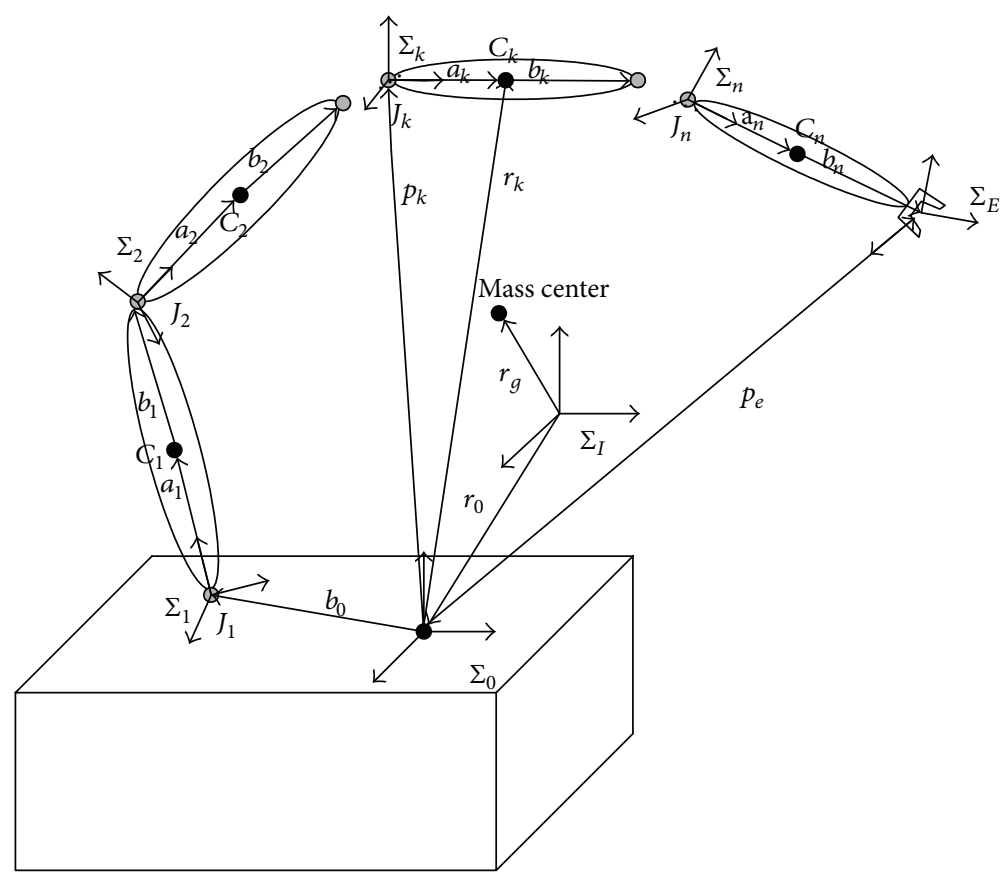

FIGURE 1: A general model of free-floating space robot. $\Sigma_{I}, \Sigma_{E}$ : the inertia frame and the end-effector frame, respectively. $\Sigma_{i}(i=1, \ldots, n)$ : the frame of link $i . C_{i}(i=1, \ldots, n)$ : the mass center position of link $i . J_{i}(i=1, \ldots, n)$ : the position of joint $i . a_{i}, b_{i}(i=1, \ldots, n)$ : the position vectors from $J_{i}$ to $C_{i}$ and $C_{i}$ to $J_{i+1}$, respectively. $r_{i}(i=1, \ldots, n)$ : the position vector of $C_{i} \cdot r_{g}$ : the position vector of the system's mass center. $p_{i}(i=1, \ldots, n)$ : the position vector of $J_{i} \cdot p_{e}$ : the position vector of the end-effector. $\Theta$ : the joint angle vector. $m_{i}(i=1, \ldots, n)$ : the mass of link $i$. $M$ : the total mass of the system. $\mathbf{E}_{3}: 3 \times 3$ identity matrix.

Combining (8) and (9), the following relationship can be obtained:

$$
\left[\begin{array}{c}
v_{e} \\
\omega_{e}
\end{array}\right]=\widehat{\mathbf{J}}_{b} \omega_{0}+\widehat{\mathbf{J}}_{m} \dot{\Theta}
$$

where

$$
\begin{gathered}
\widehat{\mathbf{J}}_{b}=\left[\begin{array}{c}
-\widetilde{\mathbf{p}}_{g e} \\
\mathbf{E}_{3}
\end{array}\right] \in \mathbf{R}^{6 \times 3}, \\
\widehat{\mathbf{J}}_{m}=\left[\begin{array}{ccc}
z_{1} \times\left(p_{e}-\widehat{p}_{1}\right) & \ldots & z_{n} \times\left(p_{e}-\widehat{p}_{n}\right) \\
z_{1} & \cdots & z_{n}
\end{array}\right] \in \mathbf{R}^{6 \times n},
\end{gathered}
$$

and $z_{i}$ is the unit vector of $z$-axis of coordinate system $i$.

When a space robot is in free-flying mode, its attitude is kept unchanged; namely, $\omega_{0}=\left[\begin{array}{ccc}0 & 0 & 0\end{array}\right]^{\mathrm{T}}$; thus

$$
\left[\begin{array}{c}
v_{e} \\
\omega_{e}
\end{array}\right]=\widehat{\mathbf{J}}_{m} \dot{\Theta}
$$

Thereby, the singularity analysis of a free-flying space robot is only related to $\widehat{\mathbf{J}}_{m}$, which only contains the virtual link parameters.
When a space robot is in free-floating manipulator, the base angular velocity can be measured by its carried sensor; namely, $\omega_{0}$ is a known term, so

$$
\left[\begin{array}{c}
v_{e} \\
\omega_{e}
\end{array}\right]-\widehat{\mathbf{J}}_{b} \omega_{0}=\widehat{\mathbf{J}}_{m} \dot{\Theta},
$$

$$
\left[\begin{array}{c}
\widehat{v}_{e} \\
\widehat{\omega}_{e}
\end{array}\right]=\widehat{\mathbf{J}}_{m} \dot{\Theta}
$$

where $\left[\begin{array}{c}\widehat{v}_{e} \\ \hat{\omega}_{e}\end{array}\right]=\left[\begin{array}{c}v_{e} \\ \omega_{e}\end{array}\right]-\widehat{\mathbf{J}}_{b} \omega_{0}$.

In the same way, the singularity analysis of a free-floating space robot is also only related to $\widehat{\mathbf{J}}_{m}$, which only contains the virtual link parameters.

According to the derivation above, it can be found that the singularity problem of space robots can be transferred to that of a ground one through the "virtual manipulator" method.

\section{Simplifying the Jacobian Matrix}

For a 7-dof space robot with the structure of Canadarm2, its axis 3 , axis 4 , and axis 5 are parallel to each other. Linkpole coordinate system based on the method of DenavitHartenberg is established in Figure 2. The relevant parameters of the space robotic system are given in Table 1, and the corresponding parameters of virtual manipulator are shown in Table 2. 


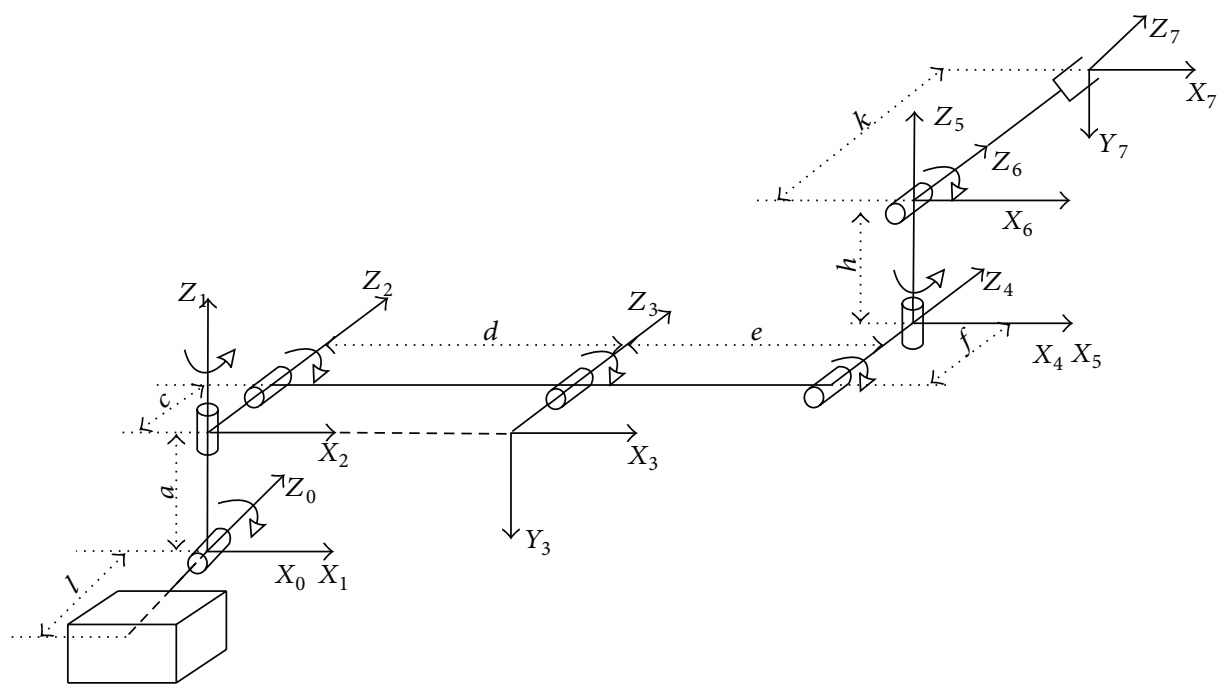

Figure 2: DH coordinate system of the studied robot.

TABLE 1: The relevant parameters of the space robotic system.

\begin{tabular}{lcccccccc}
\hline & Base & Link 1 & Link 2 & Link 3 & Link 4 & Link 5 & Link 6 & Link 7 \\
\hline Mass $/ \mathrm{kg}$ & $m_{0}$ & $m_{1}$ & $m_{2}$ & $m_{3}$ & $m_{4}$ & $m_{5}$ & $m_{6}$ & $m_{7}$ \\
$a_{i} / \mathrm{m}$ & & $a / 2$ & $c / 2$ & $d / 2$ & $e / 2$ & $f / 2$ & $h / 2$ & $k / 2$ \\
$b_{i} / \mathrm{m}$ & $l$ & $a / 2$ & $c / 2$ & $d / 2$ & $e / 2$ & $f / 2$ & $h / 2$ & $k / 2$ \\
\hline
\end{tabular}

TABLE 2: The corresponding parameters of virtual manipulator of the space robotic system.

\begin{tabular}{lcccccccc}
\hline & Base & Link 1 & Link 2 & Link 3 & Link 4 & Link 5 & Link 6 & Link 7 \\
\hline Mass/kg & $m_{0}$ & $m_{1}$ & $m_{2}$ & $m_{3}$ & $m_{4}$ & $m_{5}$ & $m_{6}$ \\
$\hat{a}_{i} / \mathrm{m}$ & & $\frac{\left(m_{0}+m_{1}\right) a}{2 M}$ & $\frac{\sum_{k=0}^{2} m_{k} c}{2 M}$ & $\frac{\sum_{k=0}^{3} m_{k} d}{2 M}$ & $\frac{\sum_{k=0}^{4} m_{k} e}{2 M}$ & $\frac{\sum_{k=0}^{5} m_{k} f}{2 M}$ & $\frac{\sum_{k=0}^{6} m_{k} h}{2 M}$ & $\frac{\sum_{k=0}^{7} m_{k} k}{2 M}$ \\
$\hat{b}_{i} / \mathrm{m}$ & $\frac{m_{0} l}{M}$ & $\frac{\left(m_{0}+m_{1}\right) a}{2 M}$ & $\frac{\sum_{k=0}^{2} m_{k} c}{2 M}$ & $\frac{\sum_{k=0}^{3} m_{k} d}{2 M}$ & $\frac{\sum_{k=0}^{4} m_{k} e}{2 M}$ & $\frac{\sum_{k=0}^{5} m_{k} f}{2 M}$ & $\frac{\sum_{k=0}^{6} m_{k} h}{2 M}$ & $\frac{\sum_{k=0}^{7} m_{k} k}{2 M}$ \\
\hline
\end{tabular}

The Jacobian matrix of the studied robot is very complicated in inertial coordinate system, making it very tough to analyze the singularity characteristics using matrix $\widehat{\mathbf{J}}_{m}$ directly; therefore, it is necessary to simplify the analytical form of $\widehat{\mathbf{J}}_{m}$. Usually, the Jacobian matrix can be simplified greatly by choosing an appropriate reference system and a proper reference point of the end-effector $[8,10]$. For the robot configuration studied in this paper, the fifth joint coordinate system $\Sigma_{4}$ in Figure 2 is chosen as the reference system $\Sigma_{\text {ref }}$ and select the intersection formed by the enlarged end-effector and the origin of $\Sigma_{\text {ref }}$ as the endeffector reference point. And the simplified Jacobian matrix can be obtained as follows:

$$
\widehat{\mathbf{J}}_{\mathrm{ref}}^{\mathrm{ref}}=\left[\begin{array}{ccccccc}
C_{2}\left(\widehat{a} C_{345}+\widehat{e} S_{5}+\widehat{d} S_{45}\right)-(\widehat{c}+\widehat{f}) S_{2} S_{345} & -(\widehat{c}+\widehat{f}) C_{345} & \hat{e} S_{5}+\widehat{d} S_{45} & \widehat{e} S_{5} & 0 & 0 & -\widehat{h} C_{6} \\
-(\widehat{c}+\hat{f}) S_{2} C_{345}+C_{2}\left(-\widehat{a} S_{345}+\widehat{e} C_{5}+\widehat{d} C_{45}\right) & (\widehat{c}+\widehat{f}) S_{345} & \widehat{e} C_{5}+\widehat{d} C_{45} & \widehat{e} C_{5} & 0 & 0 & 0 \\
-S_{2}\left(\widehat{a}-\widehat{d} S_{3}-\widehat{e} S_{34}\right) & \widehat{d} C_{3}+\widehat{e} C_{34} & 0 & 0 & 0 & 0 & -\widehat{h} S_{6} \\
C_{345} S_{2} & -S_{345} & 0 & 0 & 0 & 0 & -S_{6} \\
-S_{2} S_{345} & -C_{345} & 0 & 0 & 0 & -1 & 0 \\
C_{2} & 0 & 1 & 1 & 1 & 0 & C_{6}
\end{array}\right]
$$


where $C_{i}, S_{i}, C_{i j}, S_{i j}, C_{i j k}$, and $S_{i j k}$ represent $\operatorname{Cos}\left(\theta_{i}\right), \operatorname{Sin}\left(\theta_{i}\right)$, $\operatorname{Cos}\left(\theta_{i}+\theta_{j}\right), \operatorname{Sin}\left(\theta_{i}+\theta_{j}\right), \operatorname{Cos}\left(\theta_{i}+\theta_{j}+\theta_{k}\right)$, and $\operatorname{Sin}\left(\theta_{i}+\theta_{j}+\theta_{k}\right)$, respectively. $\widehat{a}=\left(\left(m_{0}+m_{1}\right) a\right) / M, \widehat{c}=$ $\left(\sum_{k=0}^{2} m_{k} c\right) / M, \widehat{d}=\left(\sum_{k=0}^{3} m_{k} d\right) / M, \widehat{e}=\left(\sum_{k=0}^{4} m_{k} e\right) / M, \widehat{f}=$ $\left(\sum_{k=0}^{5} m_{k} f\right) / M, \widehat{h}=\left(\sum_{k=0}^{6} m_{k} h\right) / M$, and $\widehat{k}=\left(\sum_{k=0}^{7} m_{k} k\right) /$ $M$.

In the following, the singular consistency between $\widehat{\mathbf{J}}_{\text {ref }}^{\text {ref }}$ and $\widehat{\mathbf{J}}_{m}$ is proved in detail. With respect to the reference frame, the actual end point vector $p_{e}^{\text {ref }}$ and the end reference point vector $p_{\text {ref }}^{\text {ref }}$ have the following relationship (superscript "ref" means vectors described in reference system):

$$
p_{e}^{\mathrm{ref}}=p_{\mathrm{ref}}^{\mathrm{ref}}+p_{e_{\mathrm{ref}}}^{\mathrm{ref}}
$$

where $p_{e_{\text {ref }}}^{\text {ref }}$ is the vector from the end reference point to the actual end point.

On this basis, the relationship between the end reference point velocity $v_{\text {ref }}^{\text {ref }}$ and actual end point velocity $v_{e}^{\text {ref }}$ can be expressed as

$$
v_{e}^{\mathrm{ref}}=v_{\mathrm{ref}}^{\mathrm{ref}}+\omega_{\mathrm{ref}}^{\mathrm{ref}} \times p_{e_{\mathrm{ref}}}^{\mathrm{ref}}=v_{\mathrm{ref}}^{\mathrm{ref}}-\widetilde{\mathbf{p}}_{e_{\mathrm{ref}}}^{\mathrm{ref}} \cdot \omega_{\mathrm{ref}}^{\mathrm{ref}},
$$

where $\omega_{\text {ref }}^{\text {ref }}$ is the angular velocity of the end reference point.

Since the end reference point and the actual end point attach to the same rigid body, their angular velocities are the same:

$$
\omega_{\mathrm{ref}}^{\mathrm{ref}}=\omega_{e}^{\mathrm{ref}} .
$$

Combining (17) with (18), the actual end point velocity is

$$
\dot{p}_{e}^{\mathrm{ref}}=\left[\begin{array}{c}
v_{e}^{\mathrm{ref}} \\
\omega_{e}^{\mathrm{ref}}
\end{array}\right]=\left[\begin{array}{cc}
\mathbf{E}_{3} & -\widetilde{\mathbf{p}}_{e_{\mathrm{ref}}}^{\mathrm{ref}} \\
\mathbf{O}_{3} & \mathbf{E}_{3}
\end{array}\right]\left[\begin{array}{c}
v_{\mathrm{ref}}^{\mathrm{ref}} \\
\omega_{\mathrm{ref}}^{\mathrm{ref}}
\end{array}\right]=\mathbf{J}_{e_{\mathrm{ref}}}^{\mathrm{ref}} \cdot \dot{p}_{\mathrm{ref}}^{\mathrm{ref}} \cdot
$$

Combining (12) with (19), the relationship between the original Jacobian matrix $\widehat{\mathbf{J}}_{m}$ and the simplified Jacobian matrix $\widehat{\mathbf{J}}_{\text {ref }}^{\text {ref }}$ can be represented as

$$
\widehat{\mathbf{J}}_{m}=\mathbf{J}_{\mathrm{ref}} \cdot \widehat{\mathbf{J}}_{m}^{\mathrm{ref}}=\mathbf{J}_{\mathrm{ref}} \cdot \mathbf{J}_{e_{\mathrm{ref}}}^{\mathrm{ref}} \cdot \widehat{J}_{\mathrm{ref}}^{\mathrm{ref}},
$$

where $\mathbf{J}_{\text {ref }}=\left[\begin{array}{cc}\mathbf{R}_{\text {ref }} & \mathbf{O}_{3} \\ \mathbf{O}_{3} & \mathbf{R}_{\text {ref }}\end{array}\right], \mathbf{R}_{\text {ref }}$ is the rotation matrix from $\Sigma_{\text {ref }}$ to $\Sigma_{I}$, and $\widehat{\mathbf{J}}_{m}^{\text {ref }}$ is the Jacobian matrix expressed in reference system. Because both the determinant values of $\mathbf{J}_{\text {ref }}$ and $\mathbf{J}_{e_{\text {ref }}}^{\text {ref }}$ are 1 , the singularity of $\widehat{\mathbf{J}}_{m}$ is equivalent to that of $\widehat{\mathbf{J}}_{\text {ref }}^{\text {ref }}$ The simplification will provide the foundation for robot singularity analysis in the following section.

\section{Singularity Analysis}

On the basis of the Jacobian matrix simplification, it can be found that a $3 \times 3$ zero matrix exists in $\widehat{\mathbf{J}}_{\text {ref }}^{\text {ref }}$. The zero matrix resulted from the structure with three consecutive parallel axes.

4.1. The Characteristics of the Structure of Canadarm2. For the structure of Canadarm2, which has three consecutive parallel axes, the orientations of the joint coordinate axes corresponding to three consecutive parallel axes are consistent. Without any loss of generality, take the structure of axis 3, axis 4 , and axis 5 parallel to each other as an example; the orientations of coordinate axes $z_{2}, z_{3}$, and $z_{4}$ are the same as shown in Figure 2. Since reference system $\Sigma_{\text {ref }}$ and joint coordinate system $\Sigma_{4}$ coincide with each other, the vector of axes $z_{2}, z_{3}$, and $z_{4}$ can be represented as $\{0,0,1\}^{\mathrm{T}}$ in $\Sigma_{\text {ref. }}$. Generally speaking, the first three rows of $\widehat{\mathbf{J}}_{\text {ref }}^{\text {ref }}$ are called linear velocity Jacobian matrix, and the last three rows are called angular velocity Jacobian matrix. Therefore, the angular velocity Jacobian submatrix corresponding to the three consecutive parallel axes can be obtained as follows:

$$
\mathbf{J}_{345}^{\omega}=\left[\begin{array}{lll}
0 & 0 & 0 \\
0 & 0 & 0 \\
1 & 1 & 1
\end{array}\right]
$$

As for the linear velocity Jacobian submatrix corresponding to the three consecutive parallel axes, vector $\left(p_{e}-\widehat{p}_{i}\right)$ in (10) needs to be replaced by $\left(p_{\text {ref }}^{\text {ref }}-\widehat{p}_{i}^{\text {ref }}\right)$. Both the vectors $\left(p_{\text {ref }}^{\text {ref }}-\widehat{p}_{2}^{\text {ref }}\right),\left(p_{\text {ref }}^{\text {ref }}-\widehat{p}_{3}^{\text {ref }}\right)$ are in the XOY plane of $\Sigma_{\text {ref }}$, so there are no components in $z$-axis direction. Then assume that $\left(p_{\text {ref }}^{\text {ref }}-\widehat{p}_{2}^{\text {ref }}\right)=\left\{a_{x}, a_{y}, 0\right\}^{\mathrm{T}},\left(p_{\text {ref }}^{\text {ref }}-\widehat{p}_{3}^{\text {ref }}\right)=\left\{b_{x}, b_{y}, 0\right\}^{\mathrm{T}}$, and $\left(p_{\text {ref }}^{\text {ref }}-\widehat{p}_{4}^{\text {ref }}\right)=\{0,0,0\}^{\mathrm{T}}$ are obvious. Meanwhile, considering the expression $z_{2}^{\text {ref }}=z_{3}^{\text {ref }}=z_{4}^{\text {ref }}=\{0,0,1\}^{\mathrm{T}}$, the linear velocity Jacobian submatrix corresponding to the three consecutive parallel axes is as follows:

$$
\mathbf{J}_{345}^{v}=\left[\begin{array}{ccc}
-a_{y} & -b_{y} & 0 \\
a_{x} & b_{x} & 0 \\
0 & 0 & 0
\end{array}\right]
$$

Thus, by merging (21), (22) and (23) can be constructed:

$$
\mathbf{J}_{345}=\left[\begin{array}{cc|ccc|c}
-a_{y} & a_{x} & 0 & 0 & 0 & 1 \\
-b_{y} & b_{x} & 0 & 0 & 0 & 1 \\
0 & 0 & 0 & 0 & 0 & 1
\end{array}\right]^{\mathrm{T}}
$$

Through the derivation above, it can safely draw the conclusion that a $3 \times 3$ zero matrix exists in the simplified Jacobian matrix of any robot with the structure of Canadarm2.

4.2. The Reconstruction of Jacobian Matrix. The characteristics of the structure of Canadarm 2 can be used to simplify the singularity analysis. In order to decompose the Jacobian matrix to simplify the singularity analysis, matrix $\widehat{\mathbf{J}}_{\text {ref }}^{\text {ref }}$ is reconstructed according to a novel standard, whose basic idea is to put the $3 \times 3$ zero matrix in the corner. And the new Jacobian matrix is shown as follows: 


$$
\widehat{\mathbf{J}}_{\mathrm{ref}}^{\mathrm{ref}}=\left[\begin{array}{ccccccc}
C_{2} & 0 & C_{6} & 0 & 1 & 1 & 1 \\
C_{2}\left(\widehat{a} C_{345}+\widehat{e} S_{5}+\widehat{d} S_{45}\right)-(\widehat{c}+\widehat{f}) S_{2} S_{345} & -(\widehat{c}+\widehat{f}) C_{345} & -\widehat{h} C_{6} & 0 & \widehat{e} S_{5}+\widehat{d} S_{45} & \widehat{e} S_{5} & 0 \\
-(\widehat{c}+\widehat{f}) S_{2} C_{345}+C_{2}\left(-\widehat{a} S_{345}+\widehat{e} C_{5}+\widehat{d} C_{45}\right) & (\widehat{c}+\widehat{f}) S_{345} & 0 & 0 & \widehat{e} C_{5}+\widehat{d} C_{45} & \widehat{e} C_{5} & 0 \\
-S_{2}\left(\widehat{a}-\widehat{d} S_{3}-\widehat{e} S_{34}\right) & \widehat{d} C_{3}+\widehat{e} C_{34} & -\widehat{h} S_{6} & 0 & 0 & 0 & 0 \\
C_{345} S_{2} & -S_{345} & -S_{6} & 0 & 0 & 0 & 0 \\
-S_{2} S_{345} & -C_{345} & 0 & -1 & 0 & 0 & 0
\end{array}\right] .
$$

Since the elementary transformation of matrix does not change its singularity, the singularity characteristic of the matrix $\widehat{\mathbf{J}}_{\text {ref }}^{\text {ref }}$ will be analyzed instead in the following discussion. According to (12) and (14), the relationship between the velocity of the end reference point and the joint angular velocity can be described as

$$
\dot{p}_{\text {ref }}^{\mathrm{ref}^{\prime}}=\widehat{\mathbf{J}}_{\mathrm{ref}}^{\mathrm{ref}} \cdot \dot{\theta}^{\prime},
$$

where, $\dot{p}_{\text {ref }}^{\text {ref' }}=\left\{\widehat{\omega}_{z}^{\text {ref }}, \widehat{v}_{x}^{\text {ref }}, \widehat{v}_{y}^{\text {ref }}, \widehat{v}_{z}^{\text {ref }}, \widehat{\omega}_{x}^{\text {ref }}, \widehat{\omega}_{y}^{\text {ref }}\right\}^{\mathrm{T}}$ and $\dot{\theta}^{\prime}=\left\{\dot{\theta}_{1}, \dot{\theta}_{2}\right.$, $\left.\dot{\theta}_{7}, \dot{\theta}_{6}, \dot{\theta}_{3}, \dot{\theta}_{4}, \dot{\theta}_{5}\right\}^{\mathrm{T}}$.

4.3. Singularity Analysis. On the basis of matrix reconstruction, $\widehat{\mathrm{J}}_{\mathrm{ref}}^{\text {ref }}$ can be decomposed to facilitate the singularity analysis. Considering (24) having a $3 \times 3$ zero matrix in the right bottom corner, the decomposed matrix can be written as

$$
\widehat{\mathbf{J}}_{\text {ref }}^{\text {ref' }}=\left[\begin{array}{ll}
\mathbf{J}_{11} & \mathbf{J}_{12} \\
\mathbf{J}_{21} & \mathbf{O}_{3}
\end{array}\right] .
$$

And the joint angular velocities can be obtained according to (26):

$$
\begin{aligned}
\dot{\theta}_{(5,6,7)}^{\prime} & =\left(\mathbf{J}_{12}\right)^{-1}\left[\dot{p}_{\text {ref( }(1,2,3)}^{\text {ref' }^{\prime}}-J_{11} \dot{\theta}_{(1,2,3,4)}^{\prime}\right], \\
\dot{\theta}_{(1,2,3,4)}^{\prime} & =\left(\mathbf{J}_{21}\right)^{\dagger} \dot{p}_{\text {reff(4,5,6) }}^{\text {ref' }} .
\end{aligned}
$$

Now we know that the robot singularity is only relevant to matrixes $\mathbf{J}_{12}$ and $\mathbf{J}_{21}$. In this way, singularity analysis of redundant space robots with the structure of Canadarm2 will be simplified greatly. Consequently, in the following, the singularity conditions of matrixes $\mathbf{J}_{12}$ and $\mathbf{J}_{21}$ will be discussed.

4.3.1. The Singularity Condition of Matrix $\mathbf{J}_{12}$. Because matrix $\mathbf{J}_{12}$ is a $3 \times 3$ square matrix, the singularity condition can be directly obtained through its determinant value $\widehat{d} \widehat{e} S_{4}$. Hence, Cond1: $S_{4}=0$ is the singularity condition of $\mathbf{J}_{12}$.

Substituting $S_{4}=0$ into matrix $\widehat{\mathbf{J}}_{\text {ref }}^{\text {ref }}$, we can get the base solution vector. Then, the singular direction corresponding to the singularity condition is

$$
U_{s 1}^{\mathrm{ref}}=\left\{0,-C_{5} S_{6}, S_{5} S_{6}, N_{a},-\widehat{h} N_{a}+\widehat{h} C_{5} C_{6}, 0\right\}^{\mathrm{T}},
$$

where $N_{a}=\left(-(\widehat{c}+\widehat{f}) C_{34} S_{6}+\widehat{h} S_{345} C_{5} C_{6}\right) /\left(\widehat{h} S_{345}+\widehat{e} C_{34}+\widehat{d} C_{3}\right)$.
4.3.2. The Singularity Condition of Matrix $\mathbf{J}_{21}$. Since $\mathbf{J}_{21} \in$ $\mathbf{R}^{3 \times 4}$ is not square and the determinant value expression of matrix $\mathbf{J}_{21} \cdot \mathbf{J}_{21}^{T}$ is very complicated, the singularity condition of matrix $\mathbf{J}_{21}$ will be analyzed by using its four $3 \times 3$ submatrixes [16]. All the four submatrix determinant values are listed in the following:

$$
\begin{aligned}
& \operatorname{Det}\left(J_{21(1,2,3)}\right)=S_{2} S_{6}\left(\widehat{h}+\widehat{a} C_{345}+\widehat{e} S_{5}+\widehat{d} S_{45}\right), \\
& \operatorname{Det}\left(J_{21(1,2,4)}\right)=S_{2}\left(\widehat{e} C_{5}+\widehat{d} C_{45}-\widehat{a} S_{345}\right), \\
& \operatorname{Det}\left(J_{21(1,3,4)}\right)=-S_{2} S_{6}\left(\widehat{a}+\widehat{h} C_{345}-\widehat{d} S_{3}-\widehat{e} S_{34}\right), \\
& \operatorname{Det}\left(J_{21(2,3,4)}\right)=S_{6}\left(\widehat{d} C_{3}+\widehat{e} C_{34}+\widehat{h} S_{345}\right),
\end{aligned}
$$

where $\mathbf{J}_{21(i, j, k)}$ represents the matrix composed of column $i, j, k$ of $\mathbf{J}_{21}$.

Set each determinant value of (29) to zero simultaneously, and the singularity conditions are

$$
\begin{aligned}
& \text { Cond2: } S_{2}=0, \quad S_{6}=0, \\
& \text { Cond3: } S_{2}=0, \quad \widehat{d} C_{3}+\widehat{e} C_{34}+\widehat{h} S_{345}=0, \\
& \text { Cond4: } S_{6}=0, \quad \widehat{e} C_{5}+\widehat{d} C_{45}-\widehat{a} S_{345}=0, \\
& \text { Cond5: } \widehat{d} C_{3}+\widehat{e} C_{34}+\widehat{h} S_{345}=0, \\
& \qquad \widehat{a}+\widehat{h} C_{345}-\widehat{d} S_{3}-\widehat{e} S_{34}=0 .
\end{aligned}
$$

All the conditions except Cond5 can be easily obtained. Considering the multiplication factors except $S_{2}$ and $S_{6}$ in (29), the following relationships can be found:

$$
\begin{aligned}
\widehat{h}+\widehat{a} C_{345}+\widehat{e} S_{5}+\widehat{d} S_{45}= & S_{345}\left(\widehat{d} C_{3}+\widehat{e} C_{34}+\widehat{h} S_{345}\right) \\
& +C_{345}\left(\widehat{a}+\widehat{h} C_{345}-\widehat{d} S_{3}-\widehat{e} S_{34}\right), \\
\widehat{e} C_{5}+\widehat{d} C_{45}-\widehat{a} S_{345}= & C_{345}\left(\widehat{d} C_{3}+\widehat{e} C_{34}+\widehat{h} S_{345}\right) \\
& -S_{345}\left(\widehat{a}+\widehat{h} C_{345}-\widehat{d} S_{3}-\widehat{e} S_{34}\right) .
\end{aligned}
$$

Clearly, the linear combination condition of $\widehat{h}+\widehat{a} C_{345}+$ $\widehat{e} S_{5}+\widehat{d} S_{45}=0$ and $\widehat{e} C_{5}+\widehat{d} C_{45}-\widehat{a} S_{345}=0$ is equivalent to the condition of $\widehat{d} C_{3}+\widehat{e} C_{34}+\widehat{h} S_{345}=0$ and $\widehat{a}+\widehat{h} C_{345}-\widehat{d} S_{3}-\widehat{e} S_{34}=$ 0 . Thus, Cond5 can be obtained. 
TABLE 3: The summary of examples.

\begin{tabular}{lccc}
\hline Number & Singularity condition & Joint angle & Det $\left(\widehat{\mathbf{J}}_{m} \cdot\left(\widehat{\mathbf{J}}_{m}\right)^{\mathrm{T}}\right)$ \\
\hline Cond1 & $S_{4}=0$ & $\left\{25^{\circ}, 0^{\circ}, 90^{\circ}, 0^{\circ}, 45^{\circ}, 90^{\circ}, 0^{\circ}\right\}$ & $-1.06608 \times 10^{-14}$ \\
Cond2 & $S_{2}=, S_{6}=0$ & $\left\{25^{\circ}, 0^{\circ}, 34^{\circ}, 30^{\circ}, 45^{\circ}, 0^{\circ}, 0^{\circ}\right\}$ & $-6.80933 \times 10^{-14}$ \\
Cond3 & $S_{2}=0, \widehat{d} C_{3}+\widehat{e} C_{34}+\widehat{h} S_{345}=0$ & $\left\{25^{\circ}, 0^{\circ}, 20^{\circ}, 140^{\circ},-139.1^{\circ}, 45^{\circ}, 0^{\circ}\right\}$ & $2.43766 \times 10^{-30}$ \\
Cond4 & $S_{6}=0, \widehat{e} C_{5}+\widehat{d} C_{45}-\widehat{a} S_{345}=0$ & $\left\{25^{\circ}, 10^{\circ},-132.9^{\circ}, 140^{\circ}, 20^{\circ}, 0^{\circ}, 0^{\circ}\right\}$ & $2.97193 \times 10^{-12}$ \\
Cond5 & $\widehat{d} C_{3}+\hat{e} C_{34}+\widehat{h} S_{345}=0$, & & $-1.60952 \times 10^{-16}$ \\
\hline
\end{tabular}

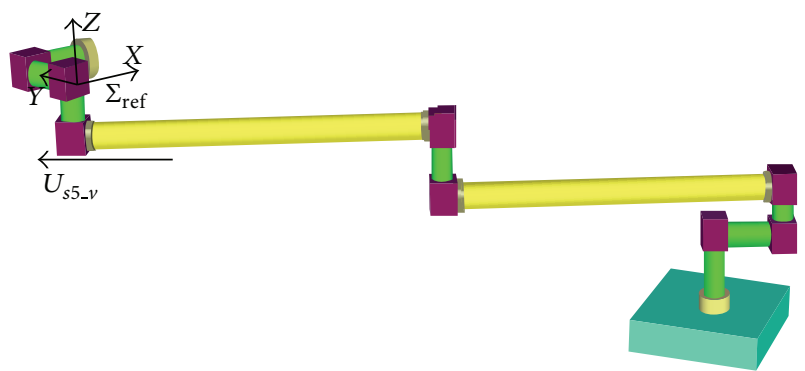

Figure 3: The singular configuration corresponding to Cond1.

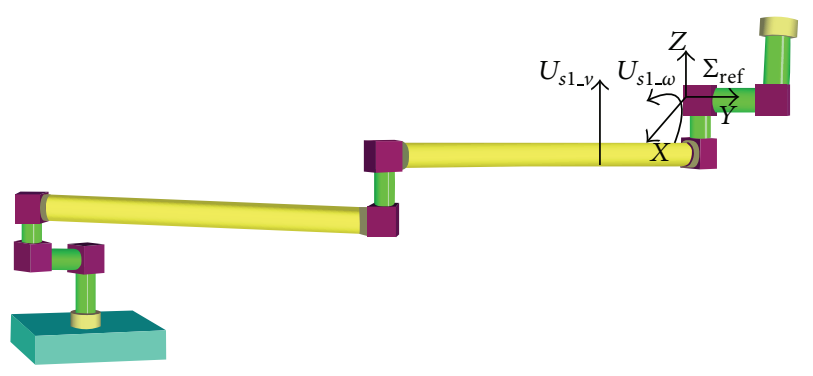

FIGURE 4: The singular configuration corresponding to Cond2.

Substituting the four singularity conditions above into

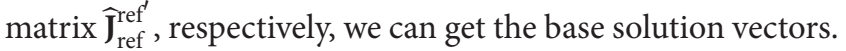
Then, the singular direction corresponding to each singularity condition is listed as

$$
\begin{aligned}
& U_{s 1}^{\mathrm{ref}}=\left\{0,0,0, S_{345}, \widehat{d} C_{3}+\widehat{e} C_{34}, 0\right\}^{\mathrm{T}}, \\
& U_{s 2}^{\mathrm{ref}}=\{0,0,0,1,-\widehat{h}, 0\}^{\mathrm{T}}, \\
& U_{s 3}^{\mathrm{ref}}=\left\{0,0,0, S_{345}, \widehat{d} C_{3}+\widehat{e} C_{34}, 0\right\}^{\mathrm{T}}, \\
& U_{s 4}^{\mathrm{ref}}=\{0,0,0,1,-\widehat{h}, 0\}^{\mathrm{T}} .
\end{aligned}
$$

\section{Simulation Results}

In order to verify the effectiveness of the singularity analysis method proposed in this paper, the following verifications are

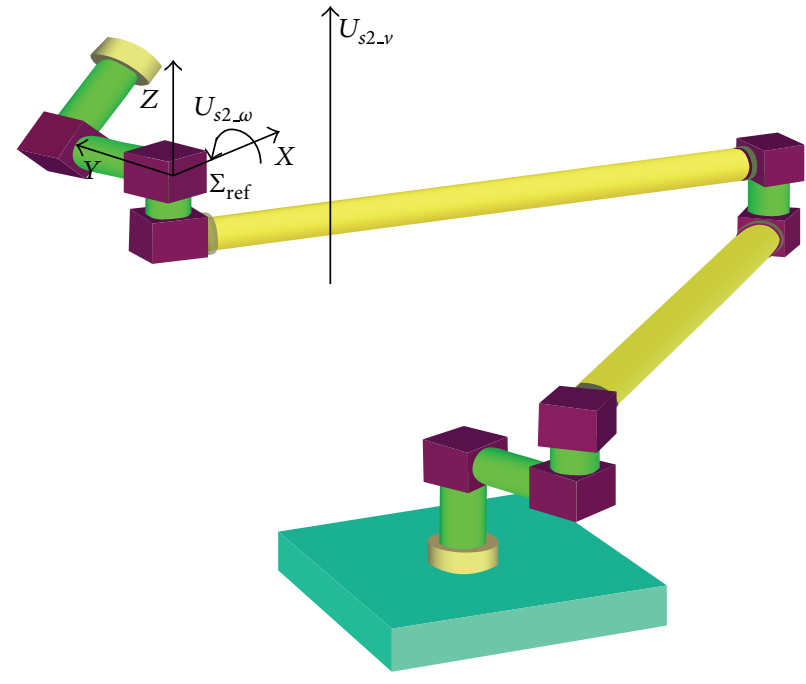

FIgURE 5: The singular configuration corresponding to Cond3.

carried out. Assign the robot parameters in Figure 2: $a=c=$ $f=h=k=l=0.5 \mathrm{~m}, d=e=2.5 \mathrm{~m}, m_{0}=1000 \mathrm{~kg}, m_{1}=$ $m_{2}=m_{5}=m_{6}=m_{7}=30 \mathrm{~kg}$, and $m_{3}=m_{4}=100 \mathrm{~kg}$. The corresponding parameters of virtual manipulator are $\widehat{a}=$ $0.38 \mathrm{~m}, \widehat{c}=0.39 \mathrm{~m}, \widehat{d}=2.15 \mathrm{~m}, \widehat{e}=2.33 \mathrm{~m}, \widehat{f}=0.48 \mathrm{~m}, \widehat{h}=$ $0.49 \mathrm{~m}$, and $\widehat{k}=0.5 \mathrm{~m}$. List five groups of robot joint angles satisfying the five singularity conditions above, respectively, and solve the corresponding determinant values of $\widehat{\mathbf{J}}_{m} \cdot\left(\widehat{\mathbf{J}}_{m}\right)^{\mathrm{T}}$. Then the relative results are shown in Table 3.

From Table 3, it can be found that all the determinant values of $\widehat{\mathbf{J}}_{m} \cdot\left(\widehat{\mathbf{J}}_{m}\right)^{\mathrm{T}}$ approach to zero. Thus, it can be judged that robot singularity happens.

According to the analytical expressions of singular directions given above, the singular configuration and singular direction corresponding to each singularity condition are shown in Figures 3, 4, 5, 6, and 7.

In the process of singularity analysis, because the simplified Jacobian matrix shows the relationship between the velocity of the end reference point and joint angular velocities in reference system $\Sigma_{\text {ref }}$, the singular direction given in Figures 3 to 7 describes the degradation direction of the end reference point expressed in reference system $\Sigma_{\text {ref }}$. 


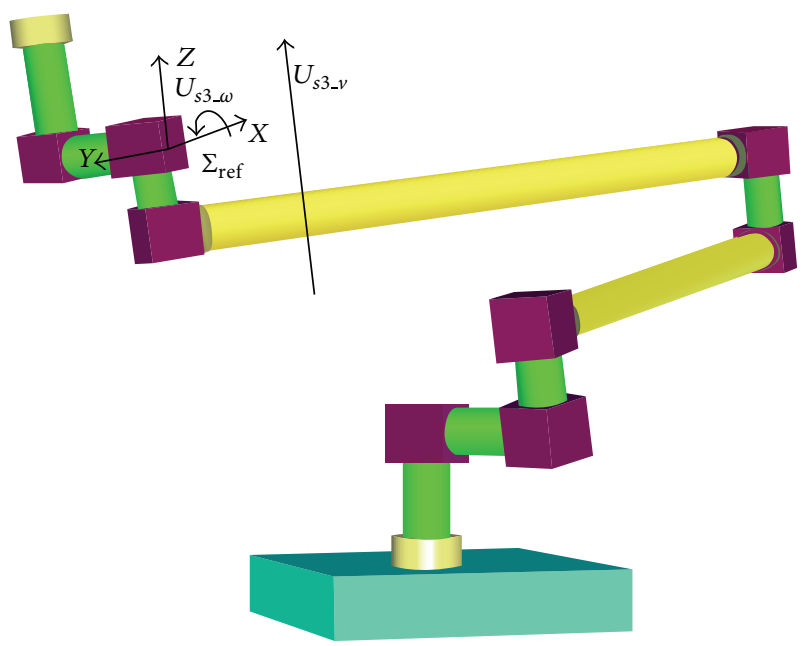

FIgURE 6: The singular configuration corresponding to Cond4.

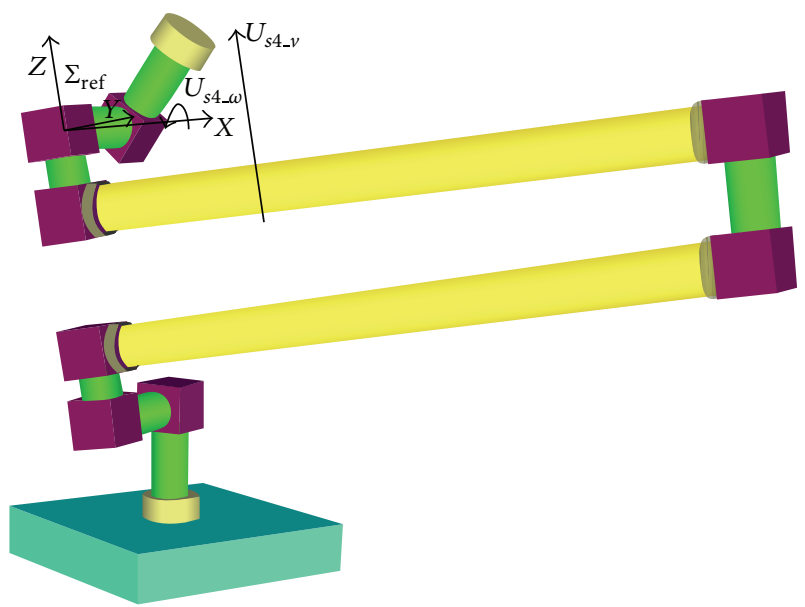

Figure 7: The singular configuration corresponding to Cond5.

The simulation results verify the effectiveness of the proposed method of singularity analysis for redundant space robots with the structure of Canadarm2.

\section{Summary and Conclusions}

A novel singularity analysis method for redundant space robots with the structure of Canadarm2 is proposed in the paper. Different from ground robots, the singularity analysis of space robots needs to consider the dynamic parameters because of the moving base. Therefore, the "virtual manipulator" method is employed to transfer the singularity problem to that of a ground one. As the generalized Jacobian matrix is very complex, it is greatly simplified by choosing a proper reference system and an end reference point and the equivalence of singularity is analyzed in detail. Then on the basis of novel reconstruction, the simplified Jacobian matrix can be decomposed to reduce the complexity of singularity analysis. Finally, through the analysis of the submatrixes, singularity conditions and singular directions of the redundant space robot with the structure of Canadarm 2 are obtained. The method proposed in the paper is effective and needs less calculation.

\section{Conflict of Interests}

The authors declare that there is no conflict of interests regarding the publication of this paper.

\section{Acknowledgments}

This project is supported by the National Key Basic Research Program of China (2013CB733000), the Specialized Research Fund for the Doctoral Program of Higher Education (20120005120004), and the Fundamental Research Funds for the Central Universities (2013PTB-00-01).

\section{References}

[1] J. Gunn, R. Blackburn, and P. J. Taylor, Forensic Psychiatry: Clinical, Legal and Ethical Issues, Butterworth-Heinemann, Oxford, UK, 1993.

[2] M. W. Spong and M. Vidyasagar, Robot Dynamics and Control, John Wiley \& Sons, 2008.

[3] D. E. Whitney, "Resolved motion rate control of manipulators and human prostheses," IEEE Transaction on Man-Machine Systems, vol. 10, no. 2, pp. 47-53, 1969.

[4] W. Chou, T. Wang, Q. Zhang, and Z. Wu, "Singularities analysis of redundant robot manipulator," Chinese Journal of Mechanical Engineering, vol. 36, no. 9, pp. 33-36, 2000.

[5] R. P. Podhorodeski, S. B. Nokleby, and J. D. Wittchen, "Resolving velocity-degenerate configurations (singularities) of redundant manipulators," in Proceedings of the Design Engineering Technical Conferences and Computers and Information in Engineering Conference, Baltimore, Md, USA, September 2000.

[6] S. B. Nokleby and R. P. Podhorodeski, "Reciprocity-based resolution of velocity degeneracies (singularities) for redundant manipulators," Mechanism and Machine Theory, vol. 36, no. 3, pp. 397-409, 2001.

[7] S. B. Nokleby and R. P. Podhorodeski, "Identifying multiDOF-loss velocity degeneracies in kinematically-redundant ,anipulators," Mechanism and Machine Theory, vol. 39, no. 2, pp. 201-213, 2004.

[8] S. B. Nokleby, "Singularity analysis of the Canadarm2," Mechanism and Machine Theory, vol. 42, no. 4, pp. 442-454, 2007.

[9] J. K. Waldron and J. Reidy, "A study of a kinematically redundant manipulator structure," in Proceedings of the IEEE International Conference on Robotics and Automation, vol. 3, pp. 1-8, IEEE, San Francisco, USA, April 1986.

[10] K. J. Waldron, S. Wang, and S. J. Bolin, "A study of the Jacobian Matrix of serial manipulators," Journal of Mechanisms, Transmissions and Automation in Design, vol. 107, no. 2, pp. 230238, 1985.

[11] F. T. Cheng, J. S. Chen, and F. C. Kung, "Study and resolution of singularities for a 7-DOF redundant manipulator," IEEE Transactions on Industrial Electronics, vol. 45, no. 3, pp. 469480, 1998. 
[12] W. $\mathrm{Xu}, \mathrm{B}$. Liang, and Y. Xu, "Practical approaches to handle the singularities of a wrist-partitioned space manipulator," Acta Astronautica, vol. 68, no. 1-2, pp. 269-300, 2011.

[13] D. Nenchev, Y. Umetani, and K. Yoshida, "Analysis of a redundant free-flying spacecraft/manipulator system," IEEE Transactions on Robotics and Automation, vol. 8, no. 1, pp. 1-6, 1992.

[14] Z. Vafa and S. Dubowsky, "On the dynamics of space manipulator using the virtual manipulator with application to path planning," The Journal of the Astronautical Science, vol. 5, no. 3, pp. 303-314, 1989.

[15] Z. Vafa and S. Dubowsky, "Kinematics and dynamics of space manipulators: the virtual manipulator approach," The International Journal of Robotics Research, vol. 9, no. 4, pp. 3-21, 1990.

[16] Robotix Associates, "A singularity analysis of the space station remote manipulator system (SSRMS)," Tech. Rep., Robotix Associates, Gainesville, Fla, USA, 1989. 


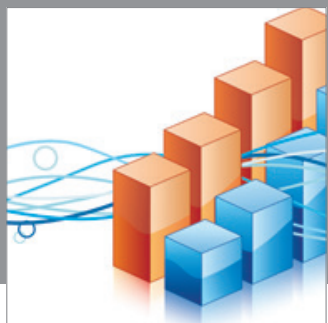

Advances in

Operations Research

mansans

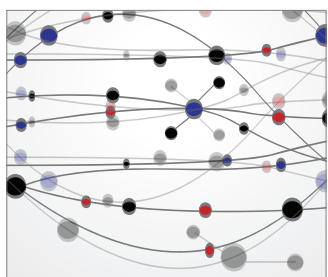

The Scientific World Journal
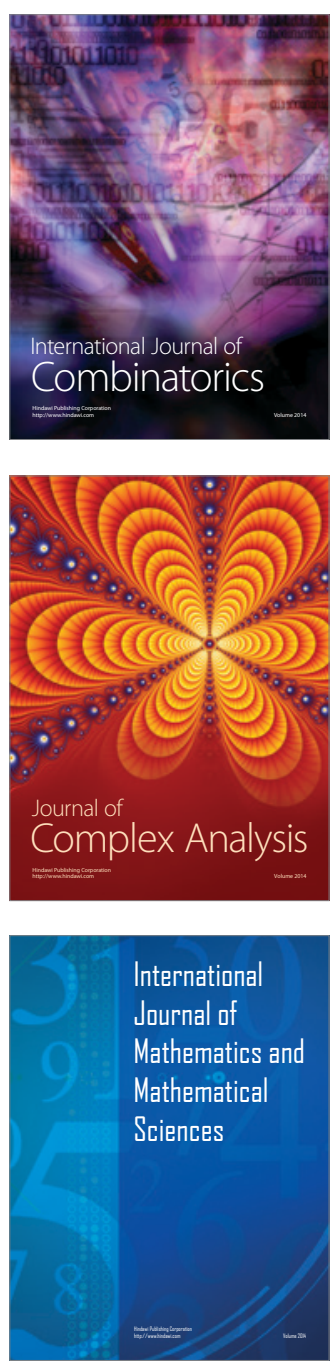
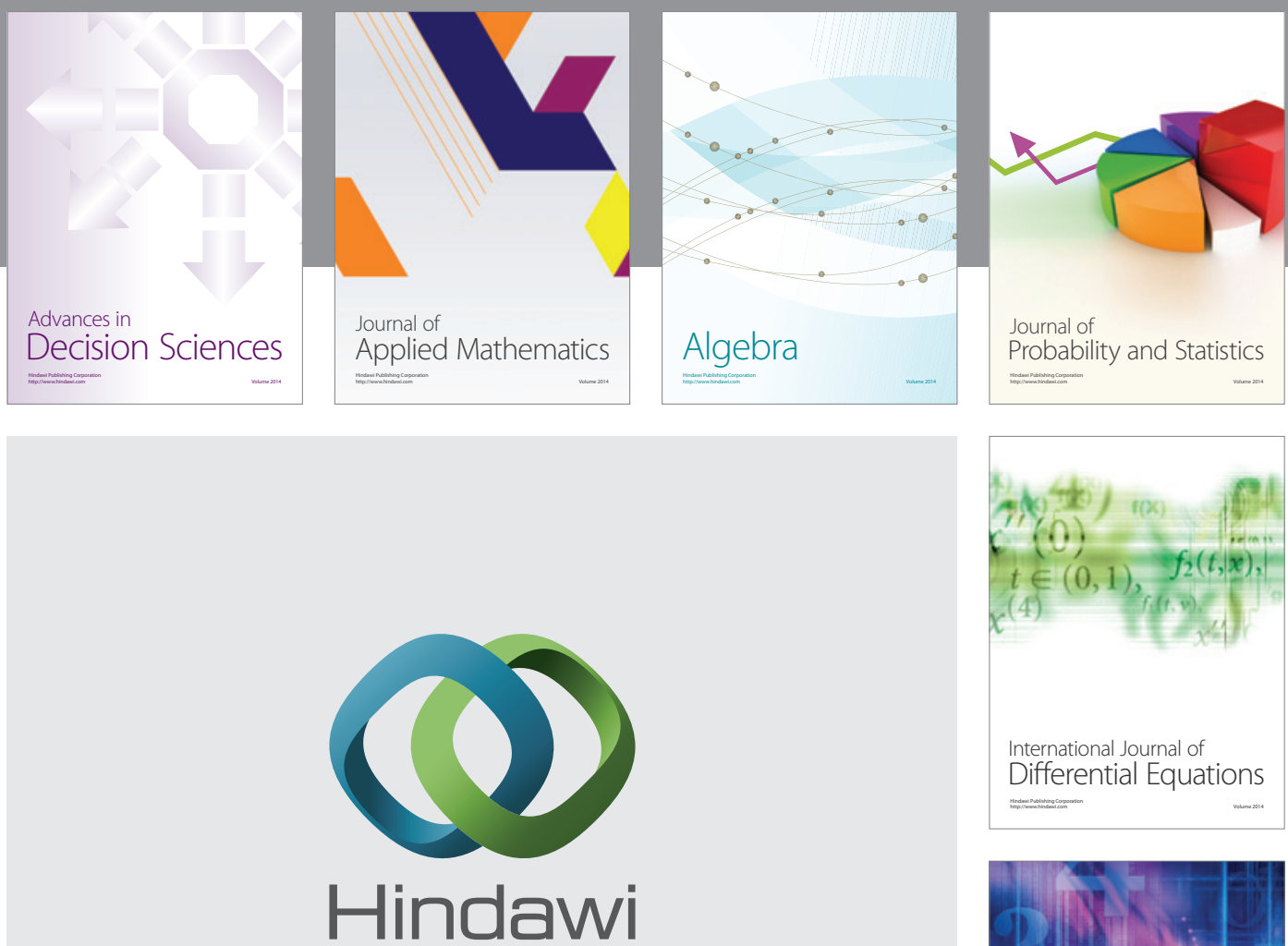

Submit your manuscripts at http://www.hindawi.com
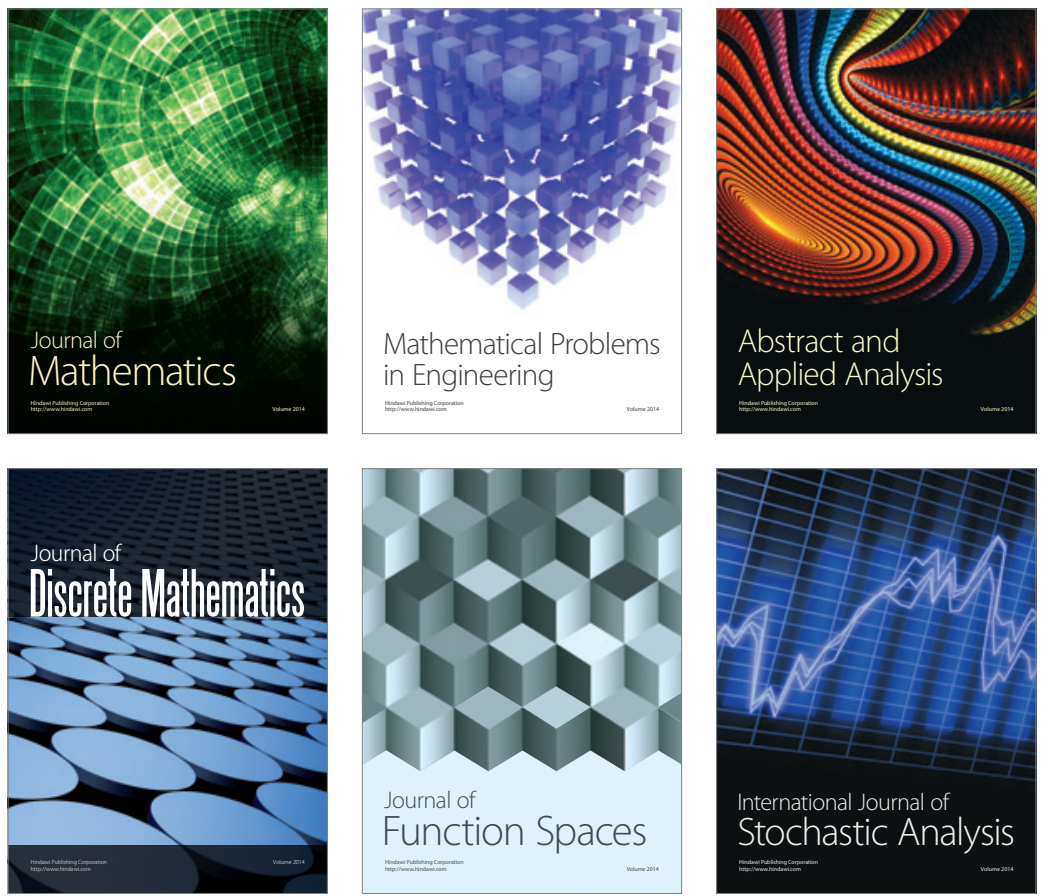

Journal of

Function Spaces

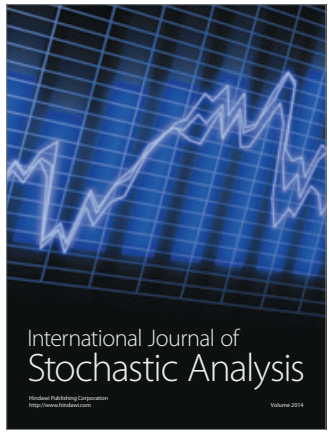

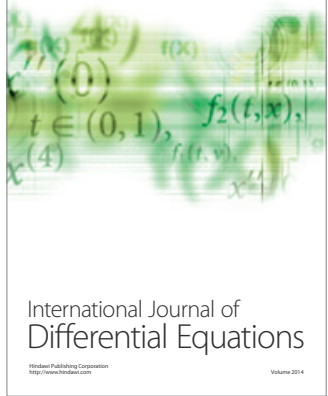
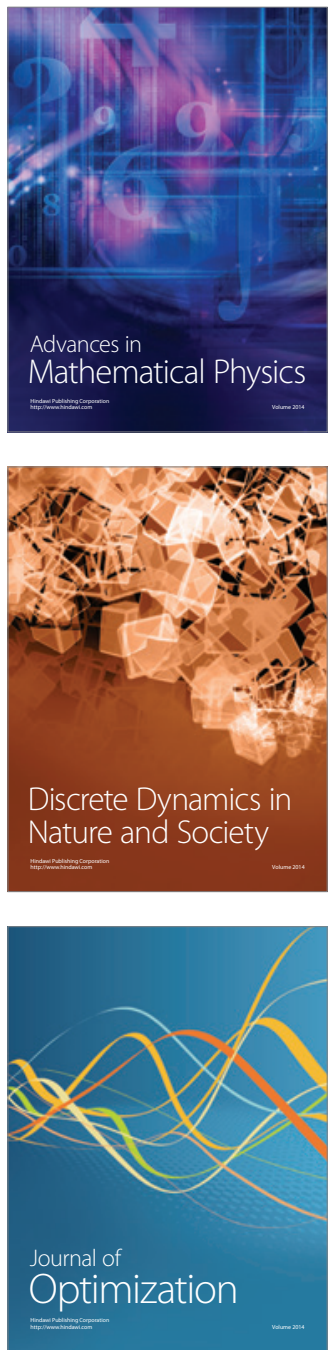